\title{
Crystal structure of metaclopromidemanganese chloride complex, $\mathrm{C}_{32} \mathrm{H}_{44} \mathrm{Cl}_{2} \mathrm{MnN}_{10} \mathrm{O}_{4} \mathrm{~S}_{4}$
}

\author{
N. K. Lokanath, M. A. Sridhar and J. Shashidhara Prasad
}

University of Mysore. Department of Studies in Physics. Manasagangothri. Mysore 570 006. India

Received May 28. 1996, CSD-No. 402509

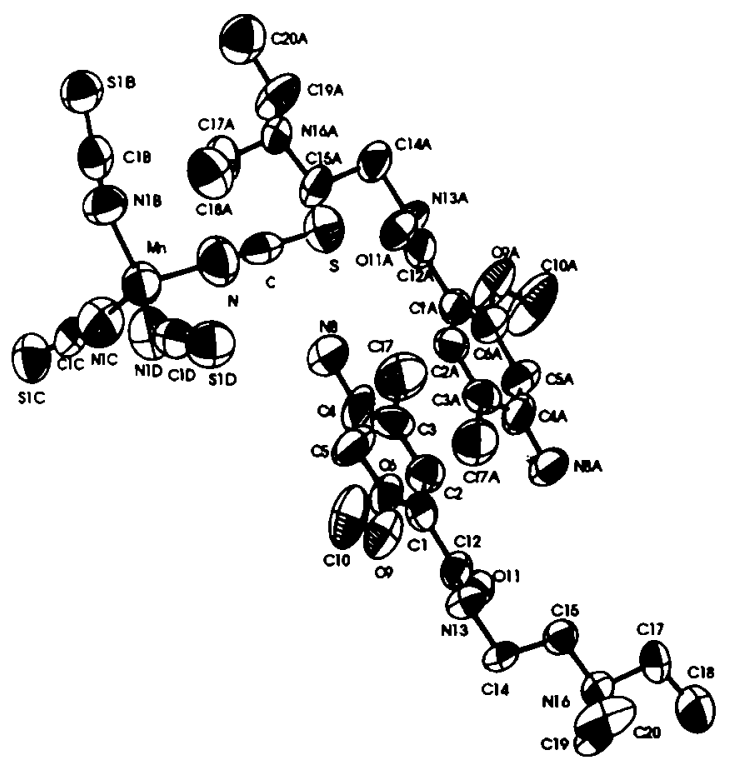

Fig. 1. ORTEP plot of the molecules.

Source of material: see ref. 1 .

The bond distances and angles do not show any large deviations. The thermal ellipsoid plot of the molecule is shown in Fig. 1. The packing of the molecules down $b$ is shown in Fig. 2. The metal and the ligands are stacked in separate layers.

$\mathrm{C}_{32} \mathrm{H}_{44} \mathrm{Cl}_{2} \mathrm{MnN}_{10} \mathrm{O}_{4} \mathrm{~S}_{4}$, orthorhombic, Pna2 1 (No. 33), $a=16.538(2) \AA, b=12.143(2) \AA, c=21.610(3) \AA, V=4339.7 \AA^{3}$, $Z=4, R(F)=0.038, R_{\mathrm{w}}\left(F^{2}\right)=0.110$.



Fig. 2. Packing of molecules down $b$.

Table 1. Parameters used for the X-ray data collection

$\begin{array}{ll}\text { Crystal: } & \text { green, rectangular, size } 0.20 \times 0.35 \times 0.35 \mathrm{~mm} \\ \text { Wavelength: } & \text { Mo } K_{\alpha} \text { radiation }(0.71069 \AA) \\ \mu: & 6.66 \mathrm{~cm}^{-1} \\ \text { Diffractometer: } & \text { Rigaku AFC7S } \\ \text { Scan mode: } & \omega / 2 \theta \\ T_{\text {measurement: }} & 293 \mathrm{~K} \\ 2 \theta_{\max }: & 50^{\circ} \\ \mathrm{N}(h k)_{\text {urique: }} & 3931 \\ \text { Criterion for } I_{\mathrm{o}}: & I_{0}>2 \sigma\left(I_{0}\right) \\ \text { N(param) } & 485 \\ \text { Programs: } & \text { SHELXS-86, SHELXIL-93 }\end{array}$

Table 2. Final atomic coordinates and displacement parameters (in $\AA^{2}$ )

\begin{tabular}{|c|c|c|c|c|c|c|c|c|c|c|}
\hline Atom & Site & $x$ & $y$ & $z$ & $U_{11}$ & $U_{22}$ & $U_{33}$ & $U_{12}$ & $U_{13}$ & $U_{23}$ \\
\hline$C\left(l^{\prime}\right)$ & $4 a$ & $-0.2576(4)$ & $-0.4093(5)$ & $1.0883(4)$ & $0.059(4)$ & $0.041(3)$ & $0.065(4)$ & $-0.001(3)$ & $0.017(3)$ & $0.002(3)$ \\
\hline$C\left(2^{\prime}\right)$ & $4 a$ & $-0.2866(5)$ & $-0.3810(6)$ & $1.0307(4)$ & $0.073(5)$ & $0.054(4)$ & $0.085(6)$ & $-0.001(4)$ & $0.015(4)$ & $0.011(4)$ \\
\hline$C\left(3^{\prime}\right)$ & $4 a$ & $-0.2702(5)$ & $-0.2807(8)$ & $1.0043(5)$ & $0.074(5)$ & $0.075(5)$ & $0.109(7)$ & $0.015(4)$ & $0.025(5)$ & $0.038(5)$ \\
\hline$C\left(4^{\prime}\right)$ & $4 a$ & $-0.2231(6)$ & $-0.2025(7)$ & $1.0338(6)$ & $0.079(5)$ & $0.051(4)$ & $0.130(9)$ & $0.003(4)$ & $0.036(6)$ & $0.026(5)$ \\
\hline$C\left(5^{\prime}\right)$ & $4 a$ & $-0.1950(6)$ & $-0.2288(7)$ & $1.0931(6)$ & $0.091(6)$ & $0.046(4)$ & $0.128(8)$ & $-0.012(4)$ & $0.045(6)$ & $-0.007(5)$ \\
\hline$C\left(6^{\prime}\right)$ & $4 a$ & $-0.2108(5)$ & $-0.3300(6)$ & $1.1206(4)$ & $0.076(5)$ & $0.041(3)$ & $0.086(5)$ & $-0.008(3)$ & $0.025(4)$ & $-0.011(4)$ \\
\hline $\mathrm{Cl}\left(7^{\prime}\right)$ & $4 a$ & $-0.3086(3)$ & $-0.2496(3)$ & $0.9314(2)$ & $0.149(3)$ & $0.142(3)$ & $0.147(3)$ & $0.010(2)$ & $0.000(2)$ & $0.089(3)$ \\
\hline$N\left(8^{\prime}\right)$ & $4 a$ & $-0.2063(6)$ & $-0.1028(7)$ & $1.0071(6)$ & $0.122(8)$ & $0.063(5)$ & $0.20(1)$ & $-0.006(5)$ & $0.037(7)$ & $0.050(6)$ \\
\hline$O\left(9^{\prime}\right)$ & $4 a$ & $-0.1844(4)$ & $-0.3593(5)$ & $1.1777(3)$ & $0.108(4)$ & $0.065(3)$ & $0.086(4)$ & $-0.040(3)$ & $0.010(4)$ & $-0.009(3)$ \\
\hline$C\left(10^{\prime}\right)$ & $4 a$ & $-0.1360(8)$ & $-0.282(1)$ & $1.2134(6)$ & $0.114(8)$ & $0.101(7)$ & $0.114(8)$ & $-0.062(7)$ & $0.003(7)$ & $-0.022(7)$ \\
\hline$O\left(11^{\prime}\right)$ & $4 a$ & $-0.3026(3)$ & $-0.5920(4)$ & $1.0733(2)$ & $0.075(3)$ & $0.050(2)$ & $0.061(3)$ & $-0.007(2)$ & $0.005(2)$ & $-0.004(2)$ \\
\hline$C\left(12^{\prime}\right)$ & $4 a$ & $-0.2777(4)$ & $-0.5214(5)$ & $1.1104(3)$ & $0.054(3)$ & $0.039(3)$ & $0.059(4)$ & $-0.002(3)$ & $0.008(3)$ & $-0.001(3)$ \\
\hline
\end{tabular}


Table 2. (Continued)

\begin{tabular}{|c|c|c|c|c|c|c|c|c|c|c|}
\hline Atom & Site & $x$ & $y$ & $z$ & $U_{11}$ & $U_{22}$ & $U_{33}$ & $U_{12}$ & $U_{13}$ & $U_{23}$ \\
\hline $\mathbf{N}\left(13^{\prime}\right)$ & $4 a$ & $-0.2702(4)$ & $-0.5454(4)$ & $1.1704(3)$ & $0.073(4)$ & $0.040(3)$ & $0.060(3)$ & $-0.013(3)$ & $0.000(3)$ & $0.001(2)$ \\
\hline$C\left(14^{\prime}\right)$ & $4 a$ & $-0.2911(4)$ & $-0.6548(5)$ & $1.1941(4)$ & $0.067(4)$ & $0.038(3)$ & $0.065(4)$ & $-0.004(3)$ & $-0.002(3)$ & $0.011(3)$ \\
\hline$C\left(15^{\prime}\right)$ & $4 a$ & $-0.3821(4)$ & $-0.6612(5)$ & $1.2017(3)$ & $0.066(4)$ & $0.042(3)$ & $0.057(4)$ & $0.004(3)$ & $0.009(3)$ & $0.010(3)$ \\
\hline$N\left(16^{\prime}\right)$ & $4 a$ & $-0.4117(3)$ & $-0.7760(4)$ & $1.2139(3)$ & $0.056(3)$ & $0.044(3)$ & $0.057(3)$ & $-0.005(2)$ & $0.004(3)$ & $0.006(2)$ \\
\hline$C\left(17^{\prime}\right)$ & $4 a$ & $-0.3764(5)$ & $-0.8274(6)$ & $1.2709(4)$ & $0.085(5)$ & $0.054(4)$ & $0.070(5)$ & $-0.013(4)$ & $0.004(4)$ & $0.020(4)$ \\
\hline$C\left(18^{\prime}\right)$ & $4 a$ & $-0.393(1)$ & $-0.7660(9)$ & $1.3299(4)$ & $0.20(1)$ & $0.086(6)$ & $0.051(4)$ & $-0.027(7)$ & $0.009(6)$ & $0.004(4)$ \\
\hline$C\left(19^{\prime}\right)$ & $4 a$ & $-0.5025(5)$ & $-0.7785(8)$ & $1.2113(5)$ & $0.054(4)$ & $0.076(6)$ & $0.107(7)$ & $0.001(4)$ & $0.000(4)$ & $0.000(5)$ \\
\hline$C\left(20^{\prime}\right)$ & $4 a$ & $-0.5377(6)$ & $-0.8913(9)$ & $1.2092(6)$ & $0.068(5)$ & $0.094(7)$ & $0.123(8)$ & $-0.020(5)$ & $-0.008(6)$ & $0.014(6)$ \\
\hline$C(1)$ & $4 a$ & $-0.3948(4)$ & $-0.0604(5)$ & $1.1020(3)$ & $0.056(3)$ & $0.047(3)$ & $0.047(3)$ & $-0.006(3)$ & $0.005(3)$ & $0.001(3)$ \\
\hline$C(2)$ & $4 a$ & $-0.3662(4)$ & $-0.0867(6)$ & $1.1603(3)$ & $0.058(4)$ & $0.050(4)$ & $0.059(4)$ & $-0.002(3)$ & $0.004(3)$ & $0.000(3)$ \\
\hline$C(3)$ & $4 a$ & $-0.3808(4)$ & $-0.1873(6)$ & $1.1862(4)$ & $0.056(4)$ & $0.061(4)$ & $0.068(5)$ & $0.009(3)$ & $0.011(3)$ & $0.016(4)$ \\
\hline$C(4)$ & $4 a$ & $-0.4263(5)$ & $-0.2666(6)$ & $1.1558(4)$ & $0.064(4)$ & $0.052(4)$ & $0.080(5)$ & $0.005(3)$ & $0.023(4)$ & $0.019(4)$ \\
\hline$C(5)$ & $4 a$ & $-0.4559(5)$ & $-0.2408(6)$ & $1.0976(4)$ & $0.086(5)$ & $0.051(4)$ & $0.067(4)$ & $-0.018(4)$ & $0.015(4)$ & $-0.002(3)$ \\
\hline$C(6)$ & $4 a$ & $-0.4408(5)$ & $-0.1377(6)$ & $1.0708(3)$ & $0.073(4)$ & $0.060(4)$ & $0.051(4)$ & $-0.015(3)$ & $0.004(3)$ & $0.002(3)$ \\
\hline$O(9)$ & $4 a$ & $-0.4697(5)$ & $-0.1105(5)$ & $1.0142(3)$ & $0.133(6)$ & $0.078(4)$ & $0.065(3)$ & $-0.062(4)$ & $-0.026(4)$ & $0.010(3)$ \\
\hline$C(10)$ & $4 a$ & $-0.521(1)$ & $-0.186(1)$ & $0.9826(6)$ & $0.21(2)$ & $0.15(1)$ & $0.092(8)$ & $-0.13(1)$ & $-0.060(9)$ & $0.033(8)$ \\
\hline$O(11)$ & $4 a$ & $-0.3478(3)$ & $0.1227(4)$ & $1.1152(2)$ & $0.082(3)$ & $0.048(2)$ & $0.052(3)$ & $-0.014(2)$ & $0.001(2)$ & $-0.007(2)$ \\
\hline$C(12)$ & $4 a$ & $-0.3747(4)$ & $0.0517(5)$ & $1.0785(3)$ & $0.053(3)$ & $0.040(3)$ & $0.054(4)$ & $-0.005(3)$ & $0.005(3)$ & $-0.004(3)$ \\
\hline$N(13)$ & $4 a$ & $-0.3814(4)$ & $0.0760(5)$ & $1.0188(3)$ & $0.088(4)$ & $0.047(3)$ & $0.054(3)$ & $-0.023(3)$ & $-0.009(3)$ & $0.002(3)$ \\
\hline$C(14)$ & $4 a$ & $-0.3577(5)$ & $0.1828(6)$ & $0.9959(4)$ & $0.078(5)$ & $0.049(4)$ & $0.065(4)$ & $-0.014(3)$ & $-0.017(4)$ & $0.013(3)$ \\
\hline$C(15)$ & $4 a$ & $-0.2656(4)$ & $0.1877(6)$ & $0.9909(3)$ & $0.074(4)$ & $0.052(4)$ & $0.049(3)$ & $0.000(3)$ & $0.005(3)$ & $0.005(3)$ \\
\hline$N(16)$ & $4 a$ & $-0.2341(4)$ & $0.3001(5)$ & $0.9741(3)$ & $0.064(3)$ & $0.056(3)$ & $0.056(3)$ & $-0.008(3)$ & $0.009(3)$ & $0.006(3)$ \\
\hline$C(17)$ & $4 a$ & $-0.2626(7)$ & $0.3322(8)$ & $0.9093(4)$ & $0.114(7)$ & $0.093(6)$ & $0.064(5)$ & $-0.024(6)$ & $0.003(5)$ & $0.022(5)$ \\
\hline$C(18)$ & $4 a$ & $-0.2421(7)$ & $0.4443(8)$ & $0.8897(5)$ & $0.115(8)$ & $0.088(6)$ & $0.086(6)$ & $0.009(6)$ & $0.011(6)$ & $0.027(5)$ \\
\hline$C(19)$ & $4 a$ & $-0.1432(6)$ & $0.3026(9)$ & $0.9794(6)$ & $0.070(6)$ & $0.088(6)$ & $0.121(9)$ & $-0.003(5)$ & $0.031(6)$ & $0.022(6)$ \\
\hline$C(20)$ & $4 a$ & $-0.1152(7)$ & $0.332(1)$ & $1.0415(8)$ & $0.074(7)$ & $0.19(1)$ & $0.16(1)$ & $0.010(8)$ & $-0.027(8)$ & $-0.03(1)$ \\
\hline $\mathbf{N}(21)$ & $4 a$ & $0.0735(6)$ & $-0.0046(7)$ & $0.7944(4)$ & $0.113(7)$ & $0.091(6)$ & $0.096(6)$ & $0.006(5)$ & $0.005(5)$ & $-0.017(5)$ \\
\hline $\mathbf{C}(22)$ & $4 a$ & $0.1157(5)$ & $-0.0323(7)$ & $0.7549(4)$ & $0.072(5)$ & $0.055(4)$ & $0.076(5)$ & $-0.006(4)$ & $-0.003(4)$ & $-0.005(4)$ \\
\hline$S(23)$ & $4 a$ & $0.1732(2)$ & $-0.0703(2)$ & $0.7000(1)$ & $0.079(1)$ & $0.094(2)$ & $0.096(2)$ & $-0.008(1)$ & $0.015(1)$ & $-0.012(1)$ \\
\hline $\mathbf{N}(24)$ & $4 a$ & $-0.1136(6)$ & $0.0393(7)$ & $0.8437(5)$ & $0.089(5)$ & $0.081(5)$ & $0.131(8)$ & $-0.006(4)$ & $-0.025(6)$ & $-0.005(5)$ \\
\hline$N(27)$ & $4 a$ & $0.0203(5)$ & $0.2400(6)$ & $0.8570(5)$ & $0.086(5)$ & $0.066(4)$ & $0.100(6)$ & $-0.001(3)$ & $-0.008(5)$ & $-0.003(4)$ \\
\hline$C(28)$ & $4 a$ & $0.0131(4)$ & $0.3331(7)$ & $0.8609(4)$ & $0.055(4)$ & $0.073(5)$ & $0.060(4)$ & $-0.004(3)$ & $-0.002(4)$ & $0.007(4)$ \\
\hline$S(29)$ & $4 a$ & $0.0050(2)$ & $0.4644(2)$ & $0.8702(2)$ & $0.097(2)$ & $0.066(1)$ & $0.103(2)$ & $0.005(1)$ & $0.005(1)$ & $0.004(1)$ \\
\hline$N(30)$ & $4 a$ & $0.0267(6)$ & $0.0333(8)$ & $0.9515(5)$ & $0.106(6)$ & $0.101(6)$ & $0.082(6)$ & $0.007(5)$ & $0.001(5)$ & $0.003(5)$ \\
\hline$C(31)$ & $4 a$ & $0.0013(6)$ & $0.0369(8)$ & $1.0007(5)$ & $0.082(6)$ & $0.070(5)$ & $0.088(7)$ & $0.007(4)$ & $-0.017(5)$ & $0.006(5)$ \\
\hline$S(32)$ & $4 a$ & $-0.0369(2)$ & $0.0429(3)$ & $1.0698(1)$ & $0.124(2)$ & $0.098(2)$ & $0.084(2)$ & $0.012(2)$ & $0.005(2)$ & $0.004(1)$ \\
\hline $\mathbf{M n}$ & $4 a$ & $0.00582(8)$ & $0.0728(1)$ & $0.86069(7)$ & $0.0752(7)$ & $0.0677(7)$ & $0.0786(8)$ & $0.0026(5)$ & $0.0000(7)$ & $-0.0104(7)$ \\
\hline
\end{tabular}

Acknowledgments. The authors thank Prof. P. G. Ramappa, Department of Chemistry, University of Mysore for kindly providing the sample. The authors also express their thanks to DST, Government of India for financial assistance under the project SP/I2/FOO/93.

\section{References}

1. Nagendrappa, G.: Studies on ion-pair complexes of d-block elements. Ph. D. Thesis. University of Mysore, India 1994.

2. Sheldrick, G. M.: Phase annealing in SHELX-90: Direct methods for larger structures. Acta Crystallogr. A46 (1990) 467-473.

3. Sheldrick, G. M.: SHELXL-93. Crystal structure refinement program. University of Göttingen, Germany 1993. 